\title{
Cell Fusion Enhances Mesendodermal Differentiation of Human Induced Pluripotent Stem Cells
}

\author{
Jie Qin, ${ }^{1,2}$ Stephanie Sontag, ${ }^{1,2}$ Qiong Lin, ${ }^{1,2}$ Saskia Mitzka, ${ }^{1,2}$ Isabelle Leisten, ${ }^{3}$ Rebekka K. Schneider, ${ }^{3}$ \\ Xiaoying Wang, ${ }^{1,2}$ Anna Jauch, ${ }^{4}$ Michael Peitz, ${ }^{5}$ Oliver Brüstle, ${ }^{5}$ Wolfgang Wagner, ${ }^{6}$ \\ Robert Chunhua Zhao, ${ }^{7,8}$ and Martin Zenke ${ }^{1,2}$
}

Human induced pluripotent stem cells (iPS cells) resemble embryonic stem cells and can differentiate into cell derivatives of all three germ layers. However, frequently the differentiation efficiency of iPS cells into some lineages is rather poor. Here, we found that fusion of iPS cells with human hematopoietic stem cells (HSCs) enhances iPS cell differentiation. Such iPS hybrids showed a prominent differentiation bias toward hematopoietic lineages but also toward other mesendodermal lineages. Additionally, during differentiation of iPS hybrids, expression of early mesendodermal markers-Brachyury (T), MIX1 Homeobox-Like Protein 1 (MIXL1), and Goosecoid (GSC) - appeared with faster kinetics than in parental iPS cells. Following iPS hybrid differentiation there was a prominent induction of NODAL and inhibition of NODAL signaling blunted mesendodermal differentiation. This indicates that NODAL signaling is critically involved in mesendodermal bias of iPS hybrid differentiation. In summary, we demonstrate that iPS cell fusion with HSCs prominently enhances iPS cell differentiation.

\section{Introduction}

$\mathrm{I}_{\mathrm{r}}$ NDUCED PLURIPOTENT STEM CELLS (iPS cells) are obtained from somatic cells by reprogramming with a specific set of transcription factors [1]. iPS cells exhibit similar properties as embryonic stem cells (ES cells) and can differentiate into cell derivatives of all three germ layers [1-5]. Importantly, iPS cells provide unique opportunities in disease modeling, drug screening, and cellular transplantation, all of which critically depend on their differentiation capacity [1-5]. However, in vitro differentiation of iPS cells has remained challenging, in particular toward mesendodermal lineages, such as hematopoietic cells, hepatocytes, and pancreatic cells [5-7]. Previous studies applied small molecules, growth factors, scaffolds, or stromal cell coculture to improve differentiation efficiency [8].

Cell fusion was used as an approach to study reprogramming of somatic cells toward pluripotency [3,9-20]. Shortly after fusion, hybrid cells acquire similar characteristics as the parental pluripotent stem cells. Hybrids show the same morphology as the parental pluripotent stem cells and exhibit similar doubling time. They express pluripotency markers, downregulate tissue-specific markers of parental somatic cells, reactivate inactive $\mathrm{X}$ chromosome of female somatic cells, and show an undifferentiated epigenetic state [14,21]. They readily differentiate into three germ layers both in vitro and in vivo [15,22]. When injected into diploid blastocysts, hybrids can generate chimeric embryos, with contribution to all the three germ layers and extraembryonic tissues [13,14,22]. Foshay et al. showed that only pluripotent stem cells can induce pluripotency in somatic cells [16]. Factors, which influence reprogramming by cell fusion, include polycomb group proteins [17], activation-induced cytidine deaminase (AID)-dependent demethylation [18], and DNA synthesis $[16,19]$. However, the differentiation capacity of hybrid cells compared with the parental pluripotent stem cells is less studied, especially in human hybrids. In particular, whether fusion impacts on the differentiation propensity of pluripotent stem cells, such as ES and iPS cells, has remained elusive.

\footnotetext{
${ }^{1}$ Department of Cell Biology, Institute for Biomedical Engineering, RWTH Aachen University Medical School, Aachen, Germany.

${ }^{2}$ Helmholtz Institute for Biomedical Engineering, RWTH Aachen University, Aachen, Germany.

${ }^{3}$ Institute of Pathology, RWTH Aachen University Medical School, Aachen, Germany.

${ }_{5}^{4}$ Institute of Human Genetics, University Heidelberg, Heidelberg, Germany.

${ }^{5}$ Institute of Reconstructive Neurobiology, Life \& Brain Center, University of Bonn and Hertie Foundation, Bonn, Germany.

${ }^{6}$ Helmholtz Institute for Biomedical Engineering, Stem Cell Biology and Cellular Engineering, RWTH Aachen University, Aachen, Germany.

${ }^{7}$ Institute of Basic Medical Sciences, Chinese Academy of Medical Sciences, School of Basic Medicine, Peking Union Medical College, Beijing, People's Republic of China.

${ }^{8}$ Center of Excellence in Tissue Engineering, Peking Union Medical College Hospital, Beijing, People's Republic of China.
} 
Here, we fused human iPS cells with hematopoietic stem cells (HSCs) from cord blood to generate pluripotent iPS/ somatic cell hybrids, in the following referred to as iPS hybrids. We hypothesized that the somatic genome in such hybrids might contribute to their differentiation potential, and thus might serve as an enhancer for iPS cell differentiation.

\section{Materials and Methods}

Detailed methods are provided as Supplementary Materials and Methods section (Supplementary Data are available online at www.liebertpub.com/scd).

\section{Cell culture and cell fusion}

CD $34^{+}$HSCs from cord blood were cultured with StemSpan Medium (STEMCELL Technologies) supplemented with $100 \mathrm{ng} / \mathrm{mL}$ SCF, $50 \mathrm{ng} / \mathrm{mL}$ FLT3 ligand, $20 \mathrm{ng} / \mathrm{mL}$ TPO, and $10 \mathrm{ng} / \mathrm{mL}$ hyper-IL-6 and infected with Puro-eGFP vector (Supplementary Fig. S1A). iPS cells were obtained from human fibroblasts by transduction with Oct4, Sox2, $\mathrm{Klf} 4$, and c-myc in retrovirus or Sendai virus vectors (Supplementary Materials and Methods section). iPS hybrids were produced from iPS cells and HSCs by polyethylene glycol fusion (Fig. 1A) and cells were seeded onto Matrigelcoated culture dishes. Puromycin selection $(4 \mu \mathrm{g} / \mathrm{mL}$; SigmaAldrich) was applied $48 \mathrm{~h}$ later and hybrid colonies were picked 1 week later and cultured on mouse embryonic fibroblast feeder. The same procedure was used to produce ES hybrids from human H9 ES cells.

\section{In vitro differentiation assay}

To induce differentiation, undifferentiated cells were dissociated with collagenase IV $\left(20-40 \mathrm{~min}, 37^{\circ} \mathrm{C}\right)$. Cell clusters were harvested and cultured in differentiation medium containing KnockOut DMEM (Invitrogen) and 20\% fetal calf serum (FCS; Lonza) in 10-cm ultra-low attachment dishes (Corning Life Sciences). On day 7 of differentiation, embryoid bodies (EBs) were plated on $0.1 \%$ gelatin-coated dishes, cultured for another 7-14 days, and then analyzed.

\section{Multicolor-fluorescence in situ hybridization analysis}

Cell samples were arrested in metaphase by KaryoMAX Colcemid Solution $\left(0.1 \mu \mathrm{g} / \mathrm{mL}\right.$, Invitrogen; $\left.2 \mathrm{~h}, 37^{\circ} \mathrm{C}\right)$. Single cells were obtained by trypsin treatment and $75 \mathrm{mM}$ of $\mathrm{KCl}$ was added. Samples were centrifuged and the cell pellet was
FIG. 1. Human induced pluripotent stem (iPS) hybrids are pluripotent. (A) Experimental design of cell fusion and iPS hybrid generation. (B) Phasecontrast images of undifferentiated iPS hybrids and parental iPS cells. Scale bar $=200 \mu \mathrm{m}$. (C) M-FISH analysis of iPS hybrid indicating a normal tetraploid karyotype 92,XXXY (above: MCK karyogram; below: inverted DAPI picture). (D) Scatter plot analysis of global gene expression of undifferentiated iPS hybrids and parental iPS cells. (E) Bidirectional hierarchical cluster analysis of gene expression in hematopoietic stem cells (HSCs), undifferentiated iPS cells, and iPS hybrids. Hybrid_1, 2, and 3 represented data from different cord blood samples. Color code: blue, low expression; yellow, high expression. (F) ChIP-quantitative polymerase chain reaction (qPCR) analysis of H3K4me3 and $\mathrm{H} 3 \mathrm{~K} 27 \mathrm{me} 3$ of OCT4, $N A N O G$, and CD45 promoters in HSCs, undifferentiated iPS cells, and iPS hybrids $(n=3)$.

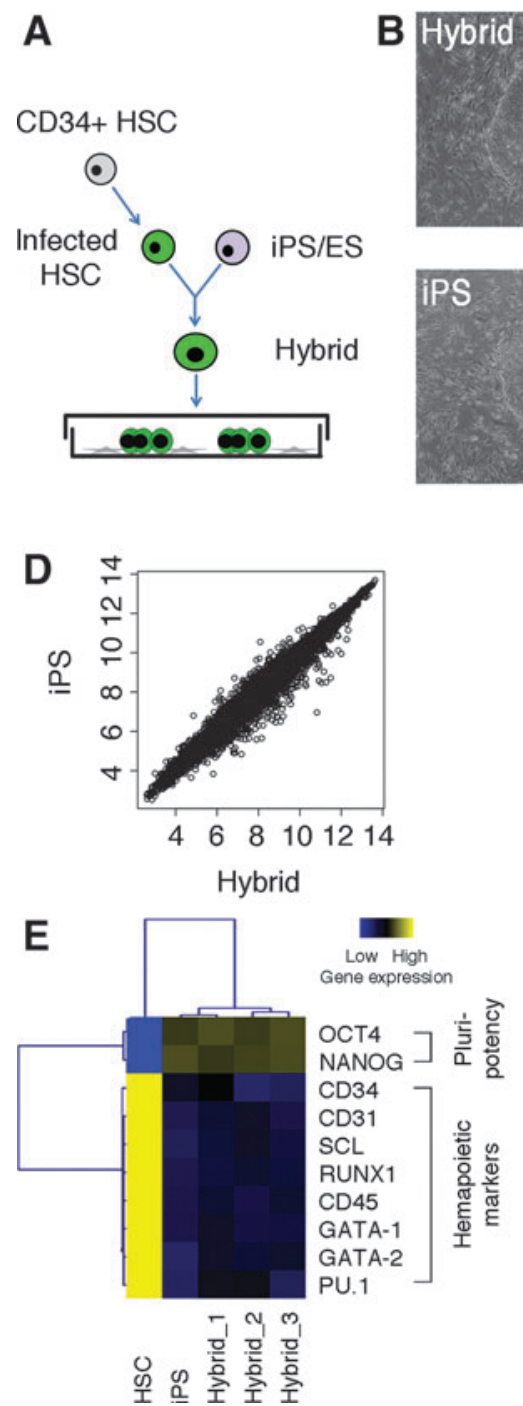

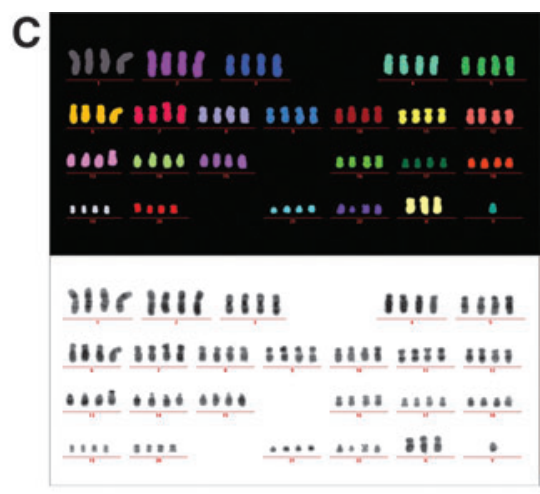

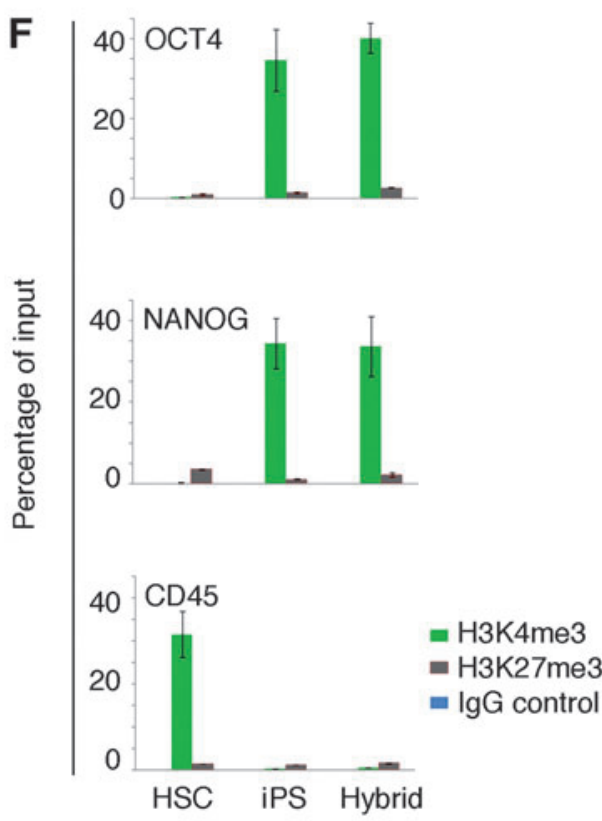


fixed with methanol/acetic acid (3:1, vol:vol). Cells were then subjected to multicolor-fluorescence in situ hybridization (MFISH) analysis. Briefly, human-chromosome-specific painting probes were combinatorially labeled using seven different fluorochromes and hybridized as previously described [23].

\section{Reverse transcription quantitative polymerase chain reaction, ChIP-quantitative polymerase chain reaction, and microarray analysis}

Total RNA was isolated and $1 \mu \mathrm{g}$ of RNA was used as template for cDNA synthesis. Quantitative polymerase chain reaction (qPCR) was carried out with StepOne RealTime PCR system (Applied Biosystems). Primer sequences are in Supplementary Table S1. The calculated threshold cycle $(\mathrm{Ct})$ value for each sample was normalized against the corresponding GAPDH value. Expression values were analyzed by using MultiExperiment Viewer (MeV, TM4.7.4; www.tm4.org/mev/).

ChIP-qPCR analysis was performed as described in Dahl and Collas [24]. Briefly, samples were fixed, sonicated, and incubated with polyclonal antibodies to H3K4me3, H3K27me3 (both Diagenode), and normal rabbit serum (Santa Cruz Biotechnology). DNA-protein samples were collected using protein-A Dynabeads (Life Technologies). DNA was prepared and subjected to qPCR analysis as described earlier. Details and primers are listed in Supplementary Materials and Methods section.

For microarray analysis, GeneChip Human Gene 1.0ST Arrays (Affymetrix) were used. Microarray data were preprocessed, normalized by RMA algorithm implemented in Affymetrix Power Tools, and subjected to hierarchical clustering analysis in R. Gene set enrichment analysis (GSEA) was performed by using predefined gene sets of different germ layers [25-27] and customized R script of GSEA. Data sets were submitted to Gene Expression Omnibus database (www .ncbi.nlm.nih.gov/geo) under accession number GSE58457.

\section{Western blotting}

Western blotting was performed according to standard procedures. Membranes were blocked and incubated with anti-pSMAD2 antibody (Ser465/467, 1:1,000 dilution; Cell Signaling Technology) or anti-ACTIN antibody (Clone AC-74, 1:5,000; Sigma-Aldrich) overnight at $4^{\circ} \mathrm{C}$. Membranes were then incubated with peroxidase-conjugated anti-rabbit or anti-mouse secondary antibody (NA934V and NA931, respectively; both 1:5,000; GE Healthcare) for $1 \mathrm{~h}$ at RT and bands were detected by chemiluminescence (ECL; GE Healthcare).

\section{Teratoma assay}

Undifferentiated cells were harvested with collagenase IV $\left(1 \mathrm{mg} / \mathrm{mL}\right.$, Invitrogen; $\left.40 \mathrm{~min}, 37^{\circ} \mathrm{C}\right)$ and $1-5 \times 10^{6}$ cells were injected subcutaneously into NOD-SCID mice. Mice were analyzed for teratoma formation 8 weeks later. All experimental procedures involving animal work were approved by local authorities in compliance with the German animal protection law.

\section{Statistical analysis}

All data were obtained from at least three independent experiments. Statistical analysis of the data was performed with unpaired Student's $t$-test. $P<0.05$ was considered statistically significant. Data are shown as mean \pm SD.

\section{Results \\ iPS hybrids show mesendodermal differentiation bias}

Hybrids from iPS cells and HSCs showed iPS cell morphology and were tetraploid (Fig. 1B, C and Supplementary Fig. S1B). iPS hybrids had similar cell proliferation rates as parental iPS cells (Supplementary Fig. S1C). They expressed the pluripotency markers OCT4, stage-specific embryonic antigen-4 (SSEA4), and tumor rejection antigens 1-60 (TRA-1-60) similar to parental iPS cells (Supplementary Fig. S1D). Further, iPS hybrids were very similar to parental iPS cells in global gene expression (Fig. 1D), including upregulation of the pluripotency markers OCT4 and NANOG and downregulation of the hematopoietic markers CD34, CD45, and RUNX1 (Fig. 1E). Accordingly, OCT4 and $N A N O G$ promoter regions of iPS hybrids showed the transcriptionally active mark $\mathrm{H} 3 \mathrm{~K} 4 \mathrm{me} 3$, similar to parental iPS cells, as determined by chromatin immunoprecipitation (ChIP) analysis (Fig. 1F). The hematopoietic marker CD45 showed the H3K4me3 mark in HSCs, which was lost in iPS hybrids. To characterize the differentiation capacity of iPS hybrids, we performed EB and teratoma assays. Tissue types of all three germ layers were found in EBs and teratomas of hybrids, indicating that iPS hybrids had three-germ-layer differentiation capacity both in vitro and in vivo (Supplementary Fig. S1E, F). Taken together, we demonstrate that iPS hybrids were pluripotent.

Interestingly, EBs of iPS hybrids showed prominent cystic structures (day 7), which were not observed or only found at low frequency in parental iPS cells (Fig. 2A). Mesodermal markers, such as CD31, CD34, HCN4, and $\alpha$-myosin heavy chain $(\alpha-M H C)$, and endodermal markers alpha-fetoprotein $(A F P)$ and albumin $(A L B)$ were more abundantly expressed in iPS hybrids compared with parental iPS cells (days 7 and 14; Fig. 2B and Supplementary Fig. S2A). Ectodermal markers, such as SRY-box containing gene 1 (SOXI) and microtubuleassociated protein 2 (MAP2), were three- to fourfold less expressed in differentiating iPS hybrids than in parental iPS cells (Fig. 2B and Supplementary Fig. S2A). Similar results were obtained by immunohistochemical staining of EB sections and teratomas (Supplementary Fig. S2B, C). Genome-wide gene expression profiling during differentiation demonstrated that iPS hybrids clustered distinctly from iPS cells (Fig. 2C). Specifically, GSEA on key transcription factors of three-germlayer differentiation showed a mesendodermal differentiation bias of iPS hybrids already at the initial stages of differentiation (days 2 and 4; Fig. 2D). Surprisingly, when we subjected iPS hybrids to neural-specific differentiation, they maintained the bias toward more mesendodermal progenies, while parental iPS cells readily differentiated into neural lineages (Fig. 2E). In addition, we found the mesendodermal differentiation bias in both early and late-passage iPS hybrids (P10 and P35, respectively; Fig. 2F).

\section{Progenies of iPS hybrids are fully functional}

We then proceeded to analyze the differentiated cell types of iPS hybrids. iPS hybrids gave rise to erythroid and 
FIG. 2. iPS hybrids show differentiation bias toward mesendoderm. (A) Embryoid body (EB) assay of iPS hybrids and parental iPS cells (day 7). Note cystic EBs in iPS hybrids. Scale bar $=200$ $\mu \mathrm{m}$. (B) Cluster analysis of gene expression in iPS hybrids and iPS cells of (A) by reverse transcription-qPCR (days 7 and 14). Hybrid_1-3 as in Fig. 1E. (C) Cluster analysis of transcriptomes of iPS hybrids and iPS cells during differentiation as in (A) (days 7 and 14). (D) Gene set enrichment analysis (GSEA) of differentiating iPS hybrids and iPS cells (days 2 and $4 ; P<0.0001$ ). (E) Bidirectional hierarchical cluster analysis of gene expression during neural differentiation. (F) Bidirectional hierarchical cluster analysis of gene expression of early and latepassage iPS hybrid_1 (P10 and $\mathrm{P} 35$, respectively) during differentiation (days 7 and 14) as in (B). Color code in (B), (E), and (F) as in Fig. 1. Data are presented as mean of three independent experiments.
A
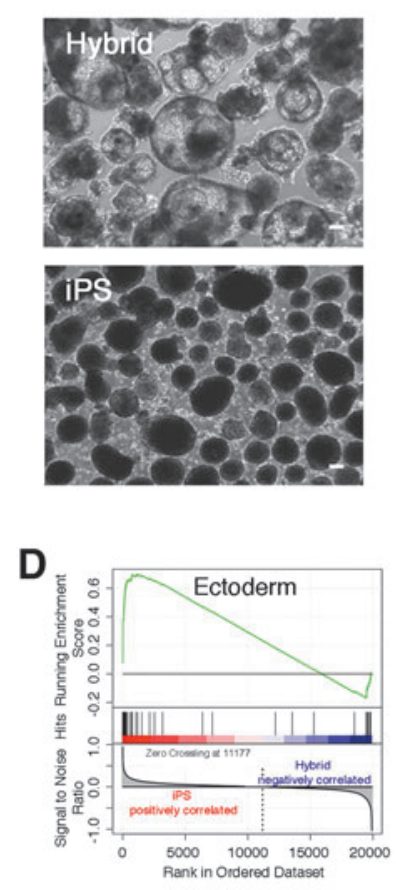

$E$

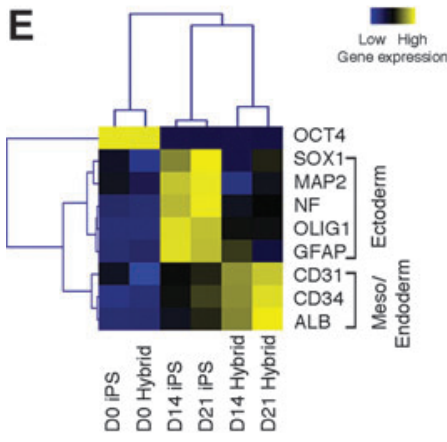

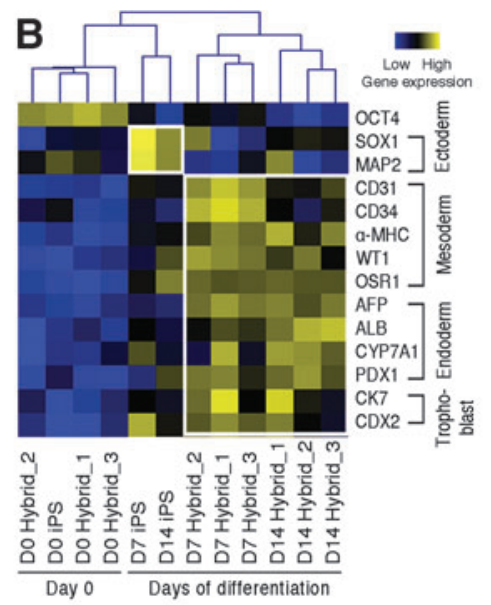

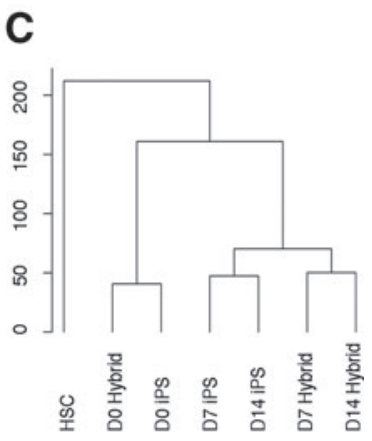

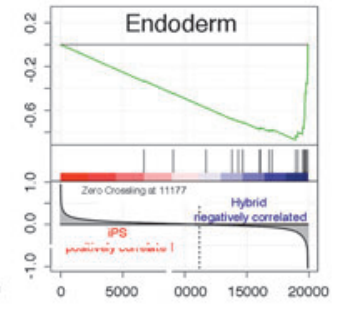

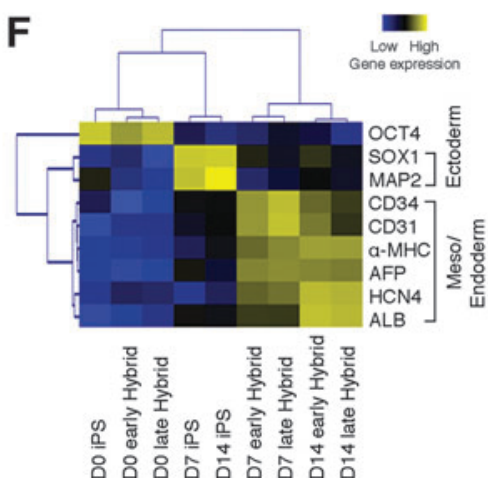

myeloid colonies under hematopoietic differentiation conditions, while colony formation of parental iPS cells was rather poor (Supplementary Fig. S3A-C and data not shown). Additionally, more $\mathrm{CD} 34^{+} \mathrm{CD} 43^{+}$and $\mathrm{CD} 34^{+} \mathrm{CD} 45^{+}$hematopoietic progenitors were obtained from iPS hybrids than from iPS cell control (Supplementary Fig. S3D, day 14). Hematopoietic progenitor cells were then differentiated into dendritic cells (DCs) with FLT3 ligand (Supplementary Fig. S3A). iPS hybrids generated more CD11 ${ }^{+}$DCs than parental iPS cells (52\% and 9\%, respectively; Supplementary Fig. S3E, day 21). The enhanced hematopoietic differentiation potential of iPS hybrids is very much in line with gene expression data of hematopoietic progenitors (CD34, CD43, and CD45) and DCs (PU.1, FLT3) by reverse transcription-qPCR (Supplementary Fig. S3F).

Further, endothelial cells derived from iPS hybrids showed the capacity of low-density lipoprotein uptake and tube formation (Supplementary Fig. S4A, B). Beating areas, indicating functional cardiomyocyte derivation, were found at a higher frequency in differentiating iPS hybrids than in parental iPS cells (day 14; Supplementary Fig. S4C and Supplementary Video). Hepatocytes derived from iPS hybrids showed the ability of urea secretion and glycogen synthesis (Supplementary Fig. S4D, E). Taking together, iPS hybrids readily differentiated into a variety of functional cells and showed a prominent differentiation bias toward mesendoderm.

iPS hybrids generated from different cord blood samples and different iPS cell lines consistently showed similar mesendodermal differentiation bias (Supplementary Fig. S5A-C). As a further control, ES hybrids were generated from human H9 ES cells and HSCs (Supplementary Fig. S6A-C). In EB assays, such ES hybrids showed impaired neural differentiation compared with parental ES cells (Supplementary Fig. S6D), as was observed for iPS hybrids. ES hybrids also showed prominent mesendodermal differentiation propensity under neural differentiation condition (Supplementary Fig. S6E). Collectively, mesendodermal differentiation propensity of hybrids is independent of HSC preparations and pluripotent cell lines.

\section{Differentiating iPS hybrids exhibit prominent and accelerated NODAL signaling}

To analyze the underlying mechanism of this mesendodermal differentiation propensity, we measured the expression 

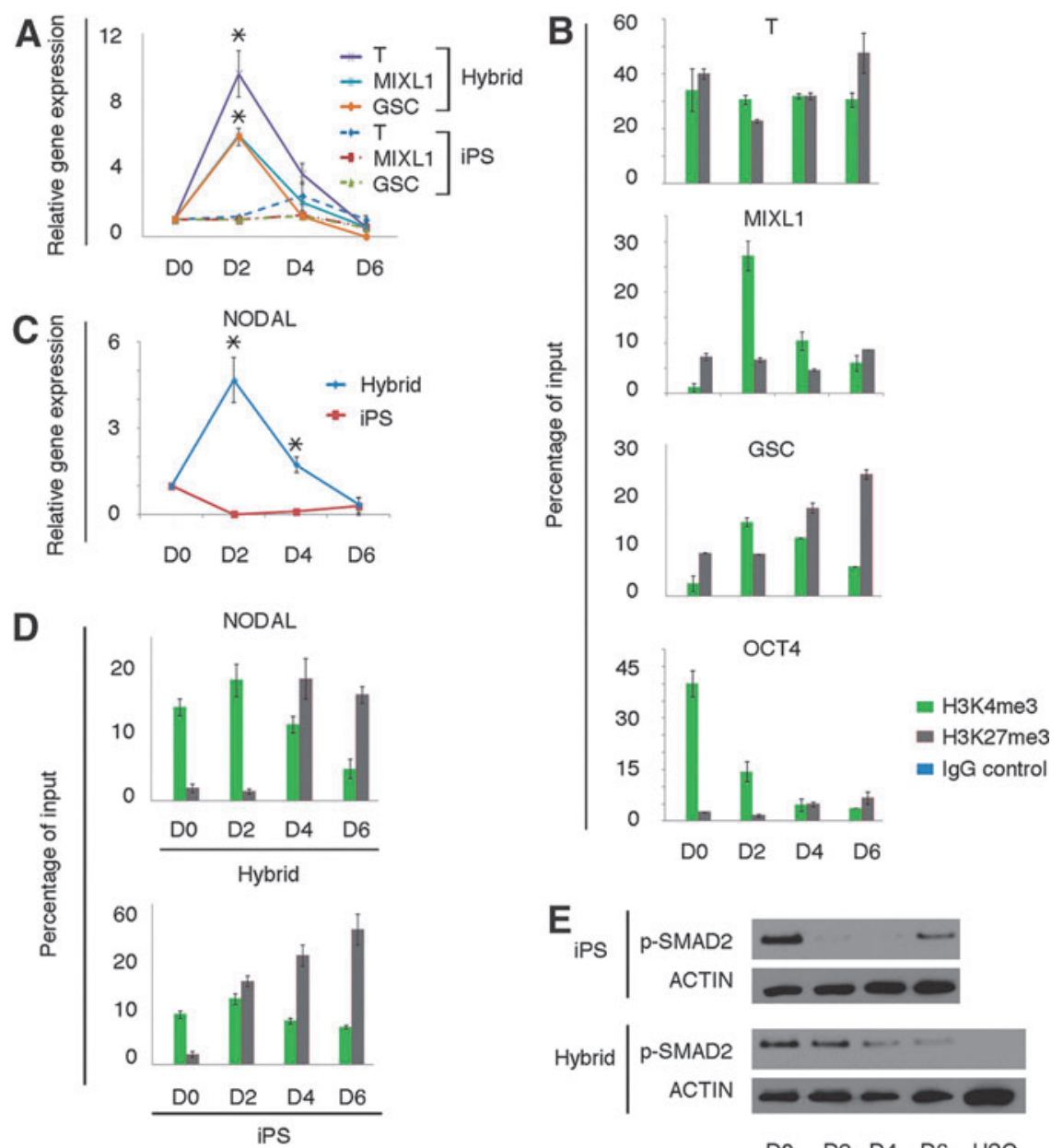

FIG. 3. Upregulation of early mesendoderm markers in iPS hybrids. (A) Expression kinetics of $T$, MIXL1, and GSC in differentiating iPS hybrids (Hybrid_1, 2, and 3) and parental iPS cells. Please note the prominent peak of $T, M I X L 1$, and GSC expression in iPS hybrids at day 2 compared with parental iPS cells $(* P<0.05)$. (B) ChIP-qPCR analysis of $\mathrm{H} 3 \mathrm{~K} 4 \mathrm{me} 3$ and $\mathrm{H} 3 \mathrm{~K} 27 \mathrm{me} 3$ in $T, M I X L 1$, and $G S C$ promoter regions of differentiating iPS hybrids as in (A) $(n=3)$. (C) NODAL expression in differentiating iPS hybrids and parental iPS cells (differential expression at days 2 and 4 , $* P<0.05$ ). (D) ChIP-qPCR analysis of $\mathrm{H} 3 \mathrm{~K} 4 \mathrm{me} 3$ and $\mathrm{H} 3 \mathrm{~K} 27 \mathrm{me} 3$ kinetics in NODAL promoter region of differentiating iPS hybrids as in (B). (E) Western blot analysis of p-SMAD2 in differentiating iPS hybrids and iPS cells as in (A). Data are presented as mean \pm SD of three independent experiments. Gene expression at day 0 was arbitrarily set 1 .

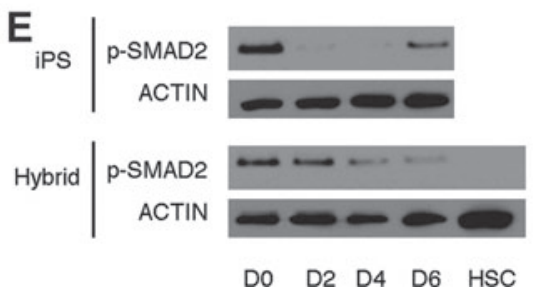

kinetics of $T, M I X L 1$, and $G S C$, which are early transcription factors of primitive streak for mesendoderm commitment [28,29]. iPS hybrids expressed all these markers at higher levels than parental iPS cells with a prominent peak at day 2 (Fig. 3A). Additionally, these expression data correlated well with an enrichment of the active mark $\mathrm{H} 3 \mathrm{~K} 4 \mathrm{me} 3$ at the $T$, MIXL1, and GSC promoters at day 2 (Fig. 3B). In parental iPS cells, there was no such enrichment at day 2 (data not shown). NODAL, an important gene for mesendodermal differentiation [29-31], showed an accelerated kinetics and a more prominent expression in differentiating iPS hybrids compared with iPS cell control (Fig. 3C). This was accompanied with an enrichment of the active mark $\mathrm{H} 3 \mathrm{~K} 4 \mathrm{me} 3$ at the NODAL promoter at day 2 (Fig. 3D). The NODAL downstream signaling protein phosphorylated-SMAD2 (pSMAD2) also occurred with accelerated kinetics at day 2 in iPS hybrids compared with parental iPS cells (Fig. 3E). Collectively, we show that ACTIVIN/NODAL signaling, which is a key component in mesendodermal commitment, is highly activated at an early stage of iPS hybrid differentiation.

To test the impact of NODAL on the mesendodermal differentiation propensity in iPS hybrids, we applied SB431542 (SB) to block ACTIVIN/NODAL/SMAD signaling. SB efficiently inhibited p-SMAD2 and the occurrence of cystic EBs (Fig. 4A-C). Primitive streak markers and lineage markers from mesendoderm were downregulated, while ectodermal markers were upregulated, indicating that the propensity of mesendodermal differentiation in iPS hybrids shifted toward ectoderm (Fig. 4D, E). Essentially the same result was obtained when applying SB in EB assays under serum-free conditions (Fig. 4F). Further, enhanced expression of primitive steak markers and NODAL signaling was also observed in other iPS hybrids (iPS_2 hybrids, Supplementary Fig. S7AC). In summary, these findings suggest that the active ACTIVIN/NODAL/SMAD signaling is a crucial component of the mesendodermal propensity in iPS hybrid differentiation.

\section{Discussion}

In this study, we demonstrate that iPS hybrids with HSCs show mesendodermal differentiation bias. Such iPS hybrids harbor enhanced differentiation capacity toward the hematopoietic lineage and also toward other mesendodermal lineages (endothelium, cardiomyocytes, and hepatocytes). Similarly, ES hybrids with HSCs also showed a mesendodermal differentiation bias. This is interesting, since neuroectoderm differentiation is considered to be the "default" pathway of in vitro differentiation of human pluripotent stem cells [32]. Thus, cell fusion of pluripotent stem cells with somatic cells provides a means to direct their differentiation propensity.

Mesoderm and endoderm originate from primitive streak and this is regulated by NODAL, WNT3, and BMP4 signaling $[29,33]$. NODAL is the major factor in primitive 
A

FIG. 4. Mesendodermal differentiation bias of iPS hybrids is blocked by ACTIVIN/NODAL inhibitor SB431542 (SB). (A) Western blot analysis of p-SMAD2 with or without SB (EB assay, day 2, 20\% FCS). (B) Phase-contrast images of EBs with or without SB as in (A) at day 7. Scale bar $=200 \mu \mathrm{m}$. (C) Frequencies of cystic EBs in differentiation culture with and without SB treatment (day 7; $* * P<0.001$ ). (D) Expression kinetics of $T, M I X L 1$, and GSC in differentiating iPS hybrids with or without SB as in (A). Please note that SB treatment efficiently blunts $T, M I X L 1$, and $G S C$ expression in iPS hybrids at day 2 $\left({ }^{*} P<0.05\right)$. (E) Bidirectional hierarchical cluster analysis of gene expression during differentiation as in (A). (F) Cluster analysis of gene expression upon neural differentiation with or without SB as in (A) but under serumfree condition. Color code in $(\mathbf{E})$ and (F) as in Fig. 1. Data are presented as mean of three independent experiments.
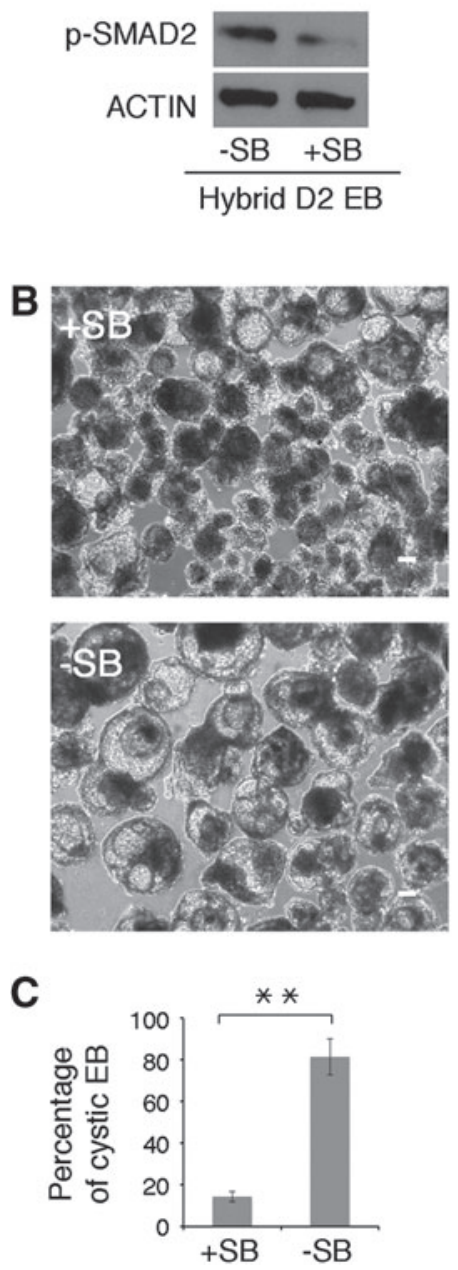
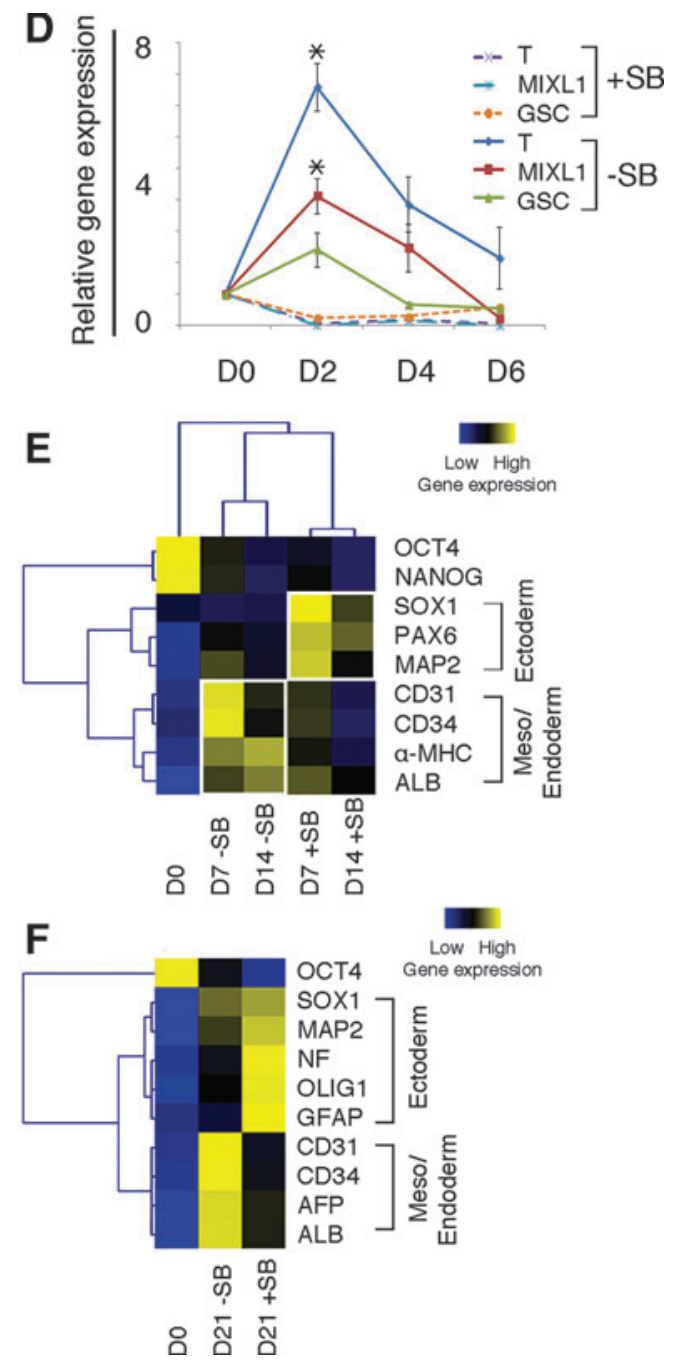

streak formation and mesendoderm development [29,30,33]. Here, we found that NODAL signaling is the main trigger of mesendodermal differentiation bias of iPS hybrids. Mesendoderm development is guided by the combined effect of several signaling pathways, including WNT3 and BMP4. Accordingly, we observed also an upregulation of WNT3 and BMP4 during differentiation in some iPS hybrid clones, which was associated with prominent mesodermal differentiation propensity (data not shown). This suggests that signaling pathways other than NODAL can participate in enhancing mesendodermal differentiation of iPS hybrids.

One interesting question is that whether the enhanced NODAL signaling in iPS hybrids is the result of "somatic memory" originating from the somatic fusion partner? Somatic memory as remnants of the somatic cell has been described in iPS cells [34,35]. Somatic memory causes a differentiation bias toward lineages of the somatic cells used for reprogramming. In addition, with continuous passaging the somatic memory of iPS cells is erased [34,36].

The differentiation bias of iPS hybrids appears to be different from the somatic memory in iPS cells. First, iPS hybrids showed a differentiation bias toward a broad range of cell types, comprising hematopoietic cells and also other mesendodermal cell types. Second, in iPS hybrids, the mesendodermal differentiation bias persisted for at least 34-40 passages, whereas the somatic memory of iPS cells vanishes with higher passage numbers (passage >12) [36]. Finally, HSCs do not express NODAL, which is the main trigger of the mesendodermal differentiation bias of iPS hybrids. As a result, the mesendodermal differentiation bias of iPS hybrids cannot simply be explained by remnants of the HSC fusion partner. So far, we cannot exclude the possibility that other unknown somatic memory factors and/or mechanisms contribute to the differentiation bias of iPS hybrids [37].

There is also the possibility that the differentiation bias observed here is a distinct trait of the $4 n$ status of the hybrids. Previous studies performed in vitro differentiation assay of hybrids to show three-germ-layer differentiation potential, but did not determine the differentiation capacity toward individual germ layers [9,14]. Experiments of mouse chimeras with cell aggregates of hybrids and normal diploid cells revealed integration of hybrid cells into a limited number of organs of the embryos (liver, heart, kidney, and gut) $[21,38]$. A competitive growth pressure between hybrid cells and diploid cells might account for this observation [39]. Alternatively, this could also be the result of the differentiation bias of the hybrid cells as observed here. 
Taken together, our results extend previous studies [3,919], where somatic cells were fused with ES cells to study reprogramming and induction of pluripotency. Here we show that fusion with somatic cells impacts on the differentiation propensity of pluripotent stem cells. In addition, our findings provide a strategy for enhancing iPS cell differentiation toward those cell types, which are notoriously difficult to obtain.

\section{Acknowledgments}

The authors would like to thank C. Becker, P. Wanek, H. Chauvistré, and H. Holtgreve-Grez for technical assistances. We also want to thank A. Offergeld and R. Sous for expert secretary assistance. This work was supported by the StemCellFactory consortium, cofunded by the European Union (European Regional Development Fund-Investing in your future) and German Federal State of North Rhine-Westphalia (M.Z., O.B., and W.W.), the Stem Cell Network NRW (W.W.), National Natural Science Foundation of China grants 30911130363 (R.C.Z.), German Federal Ministry of Education and Research (BMBF) grant no. 1315799 (O.B.), donation U. Lehman and funds from German Research Foundation (DFG) grants ZE432/5-2 and ZE432/6-1 (M.Z.).

\section{Author Disclosure Statement}

No competing financial interests exist.

\section{References}

1. Takahashi K and S Yamanaka. (2006). Induction of pluripotent stem cells from mouse embryonic and adult fibroblast cultures by defined factors. Cell 126:663-676.

2. Saha K and R Jaenisch. (2009). Technical challenges in using human induced pluripotent stem cells to model disease. Cell Stem Cell 5:584-595.

3. Yamanaka S and HM Blau. (2010). Nuclear reprogramming to a pluripotent state by three approaches. Nature 465:704-712.

4. Bellin M, MC Marchetto, FH Gage and CL Mummery. (2012). Induced pluripotent stem cells: the new patient? Nat Rev Mol Cell Biol 13:713-726.

5. Robinton DA and GQ Daley. (2012). The promise of induced pluripotent stem cells in research and therapy. Nature 481:295-305.

6. Feng Q, SJ Lu, I Klimanskaya, I Gomes, D Kim, Y Chung, GR Honig, KS Kim and R Lanza. (2010). Hemangioblastic derivatives from human induced pluripotent stem cells exhibit limited expansion and early senescence. Stem Cells 28:704-712.

7. Narsinh KH, N Sun, V Sanchez-Freire, AS Lee, P Almeida, S Hu, T Jan, KD Wilson, D Leong, et al. (2011). Single cell transcriptional profiling reveals heterogeneity of human induced pluripotent stem cells. J Clin Invest 121:12171221.

8. Mummery CL, J Zhang, ES Ng, DA Elliott, AG Elefanty and TJ Kamp. (2012). Differentiation of human embryonic stem cells and induced pluripotent stem cells to cardiomyocytes: a methods overview. Circ Res 111:344-358.

9. Cowan CA, J Atienza, DA Melton and K Eggan. (2005). Nuclear reprogramming of somatic cells after fusion with human embryonic stem cells. Science 309:13691373.
10. Hasegawa K, P Zhang, Z Wei, JE Pomeroy, W Lu and MF Pera. (2010). Comparison of reprogramming efficiency between transduction of reprogramming factors, cell-cell fusion, and cytoplast fusion. Stem Cells 28:1338-1348.

11. Yu J, MA Vodyanik, P He, Slukvin, II and JA Thomson. (2006). Human embryonic stem cells reprogram myeloid precursors following cell-cell fusion. Stem Cells 24:168 176.

12. Han DW, JT Do, L Gentile, M Stehling, HT Lee and HR Schöler. (2008). Pluripotential reprogramming of the somatic genome in hybrid cells occurs with the first cell cycle. Stem Cells 26:445-454.

13. Do JT, HW Choi, Y Choi and HR Schöler. (2011). Pluripotent hybrid cells contribute to extraembryonic as well as embryonic tissues. Stem Cells Dev 20:1063-1069.

14. Tada M, Y Takahama, K Abe, N Nakatsuji and $\mathrm{T}$ Tada. (2001). Nuclear reprogramming of somatic cells by in vitro hybridization with ES cells. Curr Biol 11:1553-1558.

15. Tat PA, H Sumer, D Pralong and PJ Verma. (2011). The efficiency of cell fusion-based reprogramming is affected by the somatic cell type and the in vitro age of somatic cells. Cell Reprogram 13:331-344.

16. Foshay KM, TJ Looney, S Chari, FF Mao, JH Lee, L Zhang, CJ Fernandes, SW Baker, KL Clift, et al. (2012). Embryonic stem cells induce pluripotency in somatic cell fusion through biphasic reprogramming. Mol Cell 46:159-170.

17. Pereira CF, FM Piccolo, T Tsubouchi, S Sauer, NK Ryan, L Bruno, D Landeira, J Santos, A Banito, et al. (2010). ESCs require $\mathrm{PRC} 2$ to direct the successful reprogramming of differentiated cells toward pluripotency. Cell Stem Cell 6:547-556.

18. Bhutani N, JJ Brady, M Damian, A Sacco, SY Corbel and HM Blau. (2010). Reprogramming towards pluripotency requires AID-dependent DNA demethylation. Nature 463: 1042-1047.

19. Tsubouchi T, J Soza-Ried, K Brown, FM Piccolo, I Cantone, D Landeira, H Bagci, H Hochegger, M Merkenschlager and AG Fisher. (2013). DNA synthesis is required for reprogramming mediated by stem cell fusion. Cell 152: 873-883.

20. Do JT and HR Schöler. (2004). Nuclei of embryonic stem cells reprogram somatic cells. Stem Cells 22:941-949.

21. Matveeva NM, AG Shilov, EM Kaftanovskaya, LP Maximovsky, AI Zhelezova, AN Golubitsa, SI Bayborodin, MM Fokina and OL Serov. (1998). In vitro and in vivo study of pluripotency in intraspecific hybrid cells obtained by fusion of murine embryonic stem cells with splenocytes. Mol Reprod Dev 50:128-138.

22. Pralong D, ML Lim, I Vassiliev, K Mrozik, N Wijesundara, P Rathjen and PJ Verma. (2005). Tetraploid embryonic stem cells contribute to the inner cell mass of mouse blastocysts. Cloning Stem Cells 7:272-278.

23. Jentsch I, J Geigl, CA Klein and MR Speicher. (2003). Seven-fluorochrome mouse M-FISH for high-resolution analysis of interchromosomal rearrangements. Cytogenet Genome Res 103:84-88.

24. Dahl JA and P Collas. (2008). A rapid micro chromatin immunoprecipitation assay (microChIP). Nat Protoc 3:10321045.

25. Koch P, T Opitz, JA Steinbeck, J Ladewig and O Brüstle. (2009). A rosette-type, self-renewing human ES cellderived neural stem cell with potential for in vitro instruction and synaptic integration. Proc Natl Acad Sci USA 106: 3225-3230. 
26. Weidgang CE, R Russell, PR Tata, SJ Kuhl, A Illing, M Müller, Q Lin, C Brunner, TM Boeckers, et al. (2013). TBX3 directs cell-fate decision toward mesendoderm. Stem Cell Rep 1:248-265.

27. Finger JH, CM Smith, TF Hayamizu, IJ McCright, JT Eppig, JA Kadin, JE Richardson and M Ringwald. (2011). The mouse Gene Expression Database (GXD): 2011 update. Nucleic Acids Res 39:D835-D841.

28. Hirst CE, ES Ng, L Azzola, AK Voss, T Thomas, EG Stanley and AG Elefanty. (2006). Transcriptional profiling of mouse and human ES cells identifies SLAIN1, a novel stem cell gene. Dev Biol 293:90-103.

29. Murry CE and G Keller. (2008). Differentiation of embryonic stem cells to clinically relevant populations: lessons from embryonic development. Cell 132:661680.

30. Xi Q, Z Wang, AI Zaromytidou, XH Zhang, LF ChowTsang, JX Liu, H Kim, A Barlas, K Manova-Todorova, et al. (2011). A poised chromatin platform for TGF-beta access to master regulators. Cell 147:1511-1524.

31. Massague J and Q Xi. (2012). TGF-beta control of stem cell differentiation genes. FEBS Lett 586:1953-1958.

32. Munoz-Sanjuan I and AH Brivanlou. (2002). Neural induction, the default model and embryonic stem cells. Nat Rev Neurosci 3:271-280.

33. Tam PP and DA Loebel. (2007). Gene function in mouse embryogenesis: get set for gastrulation. Nat Rev Genet 8: 368-381.

34. Polo JM, S Liu, ME Figueroa, W Kulalert, S Eminli, KY Tan, E Apostolou, M Stadtfeld, Y Li, et al. (2010). Cell type of origin influences the molecular and functional properties of mouse induced pluripotent stem cells. Nat Biotechnol 28:848-855.
35. Ohi Y, H Qin, C Hong, L Blouin, JM Polo, T Guo, Z Qi, SL Downey, PD Manos, et al. (2011). Incomplete DNA methylation underlies a transcriptional memory of somatic cells in human iPS cells. Nat Cell Biol 13:541-549.

36. Hussein SM, NN Batada, S Vuoristo, RW Ching, R Autio, E Narva, S Ng, M Sourour, R Hamalainen, et al. (2011). Copy number variation and selection during reprogramming to pluripotency. Nature 471:58-62.

37. Cahan P and GQ Daley. (2013). Origins and implications of pluripotent stem cell variability and heterogeneity. Nat Rev Mol Cell Biol 14:357-368.

38. Ying QL, J Nichols, EP Evans and AG Smith. (2002). Changing potency by spontaneous fusion. Nature 416:545-548.

39. Nagy A, E Gocza, EM Diaz, VR Prideaux, E Ivanyi, M Markkula and J Rossant. (1990). Embryonic stem cells alone are able to support fetal development in the mouse. Development 110:815-821.

Address correspondence to: Martin Zenke, PhD Department of Cell Biology Institute for Biomedical Engineering RWTH Aachen University Hospital Pauwelsstrasse 30 Aachen 52074 Germany

E-mail: martin.zenke@rwth-aachen.de

Received for publication March 7, 2014

Accepted after revision June 25, 2014 Prepublished on Liebert Instant Online July 8, 2014 